\title{
Deleterious ATR Gene Mutation
}

National Cancer Institute

\section{Source}

National Cancer Institute. Deleterious ATR Gene Mutation. NCI Thesaurus. Code C150148.

A change in the nucleotide sequence of the ATR gene that is associated with increased risk of disease. 